\title{
UNIFORM TWO-WEIGHT NORM INEQUALITIES FOR HANKEL TRANSFORM BOCHNER-RIESZ MEANS OF ORDER ONE
}

\author{
ÓSCAR CIAURRI, KRZYSZTOF STEMPAK, AND JUAN L. VARONA
}

\begin{abstract}
Two-weight $L^{p}$ norm inequalities, uniform with respect to the order of the involved Bessel function, are proved for the Bochner-Riesz means of the first order for the Hankel transform. Both sufficient and necessary conditions for parameters used in the two weights are determined. The proof relies on uniform pointwise asymptotic estimates for the Bessel functions that were shown by Barceló and Córdoba.
\end{abstract}

\section{INTRODUCTION}

The Hankel transform $\mathcal{H}_{\nu} f$ of a suitable function $f$ on $(0, \infty)$ is defined by

$$
\mathcal{H}_{\nu} f(x)=\int_{0}^{\infty}(x y)^{1 / 2} J_{\nu}(x y) f(y) d y, \quad x>0 .
$$

Here $\nu>-1$ is given and $J_{\nu}(x)$ denotes the Bessel function of the first kind and order $\nu$, [3]. It is known that $\left(\mathcal{H}_{\nu} \circ \mathcal{H}_{\nu}\right) f=f$ and $\left\|\mathcal{H}_{\nu} f\right\|_{2}=\|f\|_{2}$, for any $f \in$ $C_{c}^{\infty}(0, \infty)$, the space of $C^{\infty}$ functions with compact support in $(0, \infty)\left(\|\cdot\|_{p}\right.$ denotes the usual unweighted norm in $\left.L^{p}(0, \infty)\right)$. Consider the Bochner-Riesz means of order $\delta \geq 0$ for the Hankel transform $\mathcal{H}_{\nu}$ given by

$$
\begin{aligned}
S_{\nu, R}^{\delta} f(x) & =\mathcal{H}_{\nu}\left(m_{R}^{\delta} \cdot \mathcal{H}_{\nu} f\right)(x) \\
& =\int_{0}^{\infty} K_{\nu, R}^{\delta}(x, y) f(y) d y
\end{aligned}
$$

where $m_{R}^{\delta}(y)=\left(1-(y / R)^{2}\right)^{\delta}$ for $0<y<R$ and 0 otherwise, and

$$
K_{\nu, R}^{\delta}(x, y)=\int_{0}^{R} m_{R}^{\delta}(s)(x s)^{1 / 2} J_{\nu}(x s)(y s)^{1 / 2} J_{\nu}(y s) d s .
$$

Uniform boundedness, with respect to $R>0$, of $S_{\nu, R}^{\delta}$ in weighted $L^{p}$ spaces for fixed $\nu>-1$ and $\delta=0$,

$$
\left\|S_{\nu, R}^{0} f(x) x^{a}\right\|_{p} \leq C\left\|f(x) x^{a}\right\|_{p}, \quad R>0,
$$

is known to hold if either $\nu \geq-1 / 2,1<p<\infty$ and $-1 / p<a<1-1 / p$ or $-1<\nu<-1 / 2,2 /(2 \nu+3)<p<-2 /(2 \nu+1)$ and $a=0$; cf. [7] for appropriate references. The general case $\delta>0$ then follows by applying a sort of Stečkin-type multiplier theorem for the Hankel transform. Indeed, let $m(x)$ be a function of bounded variation on $(0, \infty)$. Then $(1)$ implies

$$
\left\|\mathcal{H}_{\nu}\left(m \cdot \mathcal{H}_{\nu} f\right)(x) x^{a}\right\|_{p} \leq C \max \{|m(1)|, \operatorname{Var} m\}\left\|f(x) x^{a}\right\|_{p}
$$

2000 Mathematics Subject Classification. Primary 42C10; Secondary 44A20.

Key words and phrases. Bochner-Riesz means, Hankel transform, Bessel functions, two-weight norm inequalities.

Research of the first and third authors supported by grant BFM2003-06335-C03-03 of the DGI. Research of the second author supported in part by KBN grant \# 2 P03A 03420.

THIS PAPER HAS BEEN PUBLISHED IN Tohoku Math. J. (2) 56 (2004), no. 3, 371-392. 
(with the same constant $C$ ). To show this, it is sufficient to establish the inequality

$$
\left|\int_{0}^{\infty} m(y) \mathcal{H}_{\nu} f(y) \mathcal{H}_{\nu} g(y) d y\right| \leq C \max \{|m(1)|, \operatorname{Var} m\}\left\|f(x) x^{a}\right\|_{p}\left\|g(x) x^{-a}\right\|_{p^{\prime}}
$$

for $f, g \in C_{c}^{\infty}(0, \infty)$. Define

$$
n_{\nu}(R)=\int_{0}^{R} \mathcal{H}_{\nu} f(y) \mathcal{H}_{\nu} g(y) d y, \quad R>0
$$

Then (1) gives

$$
\left|n_{\nu}(R)\right| \leq C\left\|f(x) x^{a}\right\|_{p}\left\|g(x) x^{-a}\right\|_{p^{\prime}}
$$

and an integration by parts leads to

$$
\begin{aligned}
\left|\int_{\varepsilon}^{N} m(y) \mathcal{H}_{\nu} f(y) \mathcal{H}_{\nu} g(y) d y\right| & \leq\left|m(\varepsilon) n_{\nu}(\varepsilon)\right|+\left|m(N) n_{\nu}(N)\right|+\left|\int_{\varepsilon}^{N} n_{\nu}(y) d m(y)\right| \\
& \leq C \max \{|m(1)|, \operatorname{Var} m\}\left\|f(x) x^{a}\right\|_{p}\left\|g(x) x^{-a}\right\|_{p^{\prime}} .
\end{aligned}
$$

Finally, a limit passage with $\varepsilon$ tending to 0 and $N$ tending to $\infty$ completes the argument.

Since (1), with $\delta=0$ and $R=1$ (hence also with any $R>0$ ), holds uniformly with respect to $\nu \geq 2$ (cf. [7] for appropriate comments) in the case when $4 / 3<$ $p<4$ and $-1 / p<a<1-1 / p$ the same remains valid, by applying the argument just used, for arbitrary $\delta>0$.

The present paper focuses on proving more general, uniform with respect to $\nu$, inequalities of the form

$$
\left\|S_{\nu}^{1} f(x) x^{a}(1+x)^{b-a}\right\|_{p} \leq C\left\|f(x) x^{A}(1+x)^{B-A}\right\|_{p} ;
$$

we simplify the notation by writing $S_{\nu}^{1}$ and $K_{\nu}^{1}$ in place of $S_{\nu, 1}^{1}$ and $K_{\nu, 1}^{1}$.

Theorem 1. Let $\alpha>-1$ and $1 \leq p \leq \infty$. Then

$$
\left\|S_{\nu}^{1} f(x) x^{a}(1+x)^{b-a}\right\|_{p} \leq C\left\|f(x) x^{A}(1+x)^{B-A}\right\|_{p}
$$

with a constant $C=C(p, \alpha, a, b, A, B)$ independent of $\nu \geq \alpha$ and $f$ if and only if $a, b, A, B, \alpha$ and $p$ satisfy the conditions

(3) $a>-1 / p-(\alpha+1 / 2)(\geq$ if $p=\infty), \quad A<1-1 / p+(\alpha+1 / 2)(\leq$ if $p=1)$,

$$
b<2-1 / p(\leq \text { if } p=\infty), \quad B>-1-1 / p(\geq \text { if } p=1),
$$

$$
b \leq B
$$

Moreover, only $\nu=\alpha$ in (2) is required to prove necessity of (3), (4) and (5).

It is tacitly understood that when assuming (2) to hold and then proving the necessity of $(3),(4)$ and (5) only such functions $f$ are admitted for which $S_{\alpha}^{1} f(x)$ is well defined for (almost) all $x>0$, i.e., $\int_{0}^{\infty}\left|K_{\alpha}^{1}(x, y) f(y)\right| d y<\infty$ for $x>0$. Clearly, if $f$ is compactly supported in $(0, \infty)$, say in $[c, d], 0<c<d<\infty$, and $\int_{c}^{d}|f(y)| d y<\infty$, then $\int_{0}^{\infty}\left|K_{\nu}^{1}(x, y) f(y)\right| d y<\infty$, since, with given $x>0$, $K_{\nu}^{1}(x, y)$ is a continuous function of $y>0$. But if we assume $\int_{0}^{\infty} \mid f(x) x^{A}(1+$ $x)\left.^{B-A}\right|^{p} d x<\infty$ with $A<1-1 / p+(\alpha+1 / 2)(\leq$ if $p=1)$ and $B>-1-1 / p(\geq$ if $p=1)$, then $S_{\nu}^{1} f(x)$ is also well-defined for $\nu \geq \alpha$ and $x>0$. This easily follows, by using Hölder's inequality and the estimates $K_{\nu}^{1}(x, y)=O\left(y^{\nu+1 / 2}\right), y \rightarrow 0^{+}$, and $K_{\nu}^{1}(x, y)=O\left(y^{-2}\right), y \rightarrow \infty$. The first estimate follows from the integral representation of $K_{\nu}^{1}(x, y)$ and the asymptotic

$$
J_{\nu}(t)=O\left(t^{\nu}\right), \quad t \rightarrow 0^{+},
$$


the second is a consequence of the Campbell-type representation of $K_{\nu}^{1}(x, y)$ : cf. (26) and follow the argument in the proof of Lemma 2 (now, with $x$ and $\nu$ fixed!). Therefore the second parts of (3) and (4) should be considered as "natural" assumptions. An analysis also shows that the first conditions in (3) and (4) are dual to their right counterparts.

It is instructive to compare the assumptions (3), (4) and (2) of Theorem 1 with those of Theorem 1.1 of [7] (partial sums case). The conditions on $a$ and $A$ are exactly the same; this is probably explained by the fact that the kernels $K_{\nu}^{1}(x, y)$ and $K_{\nu}^{0}(x, y)$ have the same behaviour in the neighbourhood of $(0,0)$. The conditions on $b$ and $B$ are now relaxed (by one from both sides) when compared with those from Theorem 1.1 of [7]. The most important difference is with the condition on the relation between $b$ and $B$; it becomes now very simple when compared with that of Theorem 1.1 of [7]. Furthermore, the uniformity of (2) for large $\nu$ does not imply additional restrictions: the condition $b \leq B$ is sufficient for (2) to hold for $\nu \geq \alpha$ as well as for the single $\nu=\alpha$.

In [7] we compared a similar result proved there for partial sum operators (the case of $\delta=0$ ) with Muckenhoupt's result [4] proved for partial sum operators for expansions with respect to the system of Laguerre functions

$$
\psi_{n}^{\alpha}(x)=\left(\frac{2 \Gamma(n+1)}{\Gamma(n+\alpha+1)}\right)^{1 / 2} e^{-x^{2} / 2} x^{\alpha+1 / 2} L_{n}^{\alpha}\left(x^{2}\right),
$$

which form a complete orthonormal system in $L^{2}(0, \infty)$ (for a motivation leading to such a comparition see again [7]).

It seems worthy to compare our present result with that of Muckenhoupt and Webb [5] specified to the first order Cesàro means and reformulated for expansions with respect to $\left\{\psi_{n}^{\alpha}\right\}_{n=0}^{\infty}$,

$$
\sigma_{N}^{(\alpha, 1)}(f, x)=\frac{1}{N+1} \sum_{n=0}^{N}(N+1-n)\left\langle f, \psi_{n}^{\alpha}\right\rangle \psi_{n}^{\alpha}(x) .
$$

An earlier result of Poiani, [6], admitted the case $a=A$ and $b=B$ only and did not include some possibilities.

Theorem ([5], Theorem (2.29)). Let $\alpha>-1$ and $1 \leq p \leq \infty$. Then

$$
\sup _{N \geq 0}\left\|\sigma_{N}^{(\alpha, 1)}(f, x) x^{a}(1+x)^{b-a}\right\|_{p} \leq C\left\|f(x) x^{A}(1+x)^{B-A}\right\|_{p},
$$

holds with $C=C(p, \alpha, a, b, A, B)$ independent of $f$ if and only if $a, b, A, B$ and $\alpha$ satisfy the conditions

$$
\text { (9) } A \leq a, \quad A \leq 1-1 / p+1 / 2, \quad A<1-1 / p+(\alpha+1 / 2)(\leq \text { if } p=1) \text {, }
$$

(10) $a+B \geq \min \{-3-2 /(3 p),-2-2 / p\}, \quad A+b \leq \min \{4-2 / p, 11 / 3-2 /(3 p)\}$,

$$
\begin{gathered}
b \leq \min \{3-1 / p, 8 / 3+1 /(3 p)\}, \quad B \geq \max \{-3+1 /(3 p),-2-1 / p\}, \\
b \leq B+\min \{0,3-4 /(3 p), 5 / 3+4 /(3 p)\} .
\end{gathered}
$$

The last parts of the assumptions (8) and (9) are identical with our assumptions on $a$ and $A$, cf. (3). This is caused by the fact that the kernel $K_{\alpha}^{1}(x, y)$ and the kernels that correspond to $\sigma_{N}^{(\alpha, 1)}$ have the same behaviour for small $x$ and $y$. To see this, one has to compare the result of Lemma 1 below with the estimate (2.3) of [5] (because of the reformulation mentioned above, in (2.3) of [5] one has to take $x^{2}, y^{2}$ in place of $x, y$, and then multiply both sides by $(x y)^{1 / 2}$ ). In general, however, the 
assumptions (8)-(12) from the Laguerre case are much more involved than those from Theorem 1. This is again explained by more complicated nature of estimates of the first order Cesàro kernels in the Laguerre case (cf. (2.3), (2.4) and (2.2) in [5] with necessary modifications) when compared with the estimates contained in Lemmas 1-4 below.

\section{Preliminaries}

We start with writing an exact expression for the kernel

$$
K_{\nu}^{1}(x, y)=\sqrt{x y} \int_{0}^{1} s\left(1-s^{2}\right) J_{\nu}(x s) J_{\nu}(y s) d s
$$

that corresponds to the Bochner-Riesz mean $S_{\nu}^{1}$. We use the notation

$$
u=x^{2}-y^{2}, \quad v=x^{2}+y^{2}
$$

and

$$
\begin{array}{ll}
F_{1}(x, y)=\sqrt{x y} J_{\nu}(x) J_{\nu}(y), & F_{2}(x, y)=\sqrt{x y} J_{\nu}^{\prime}(x) J_{\nu}(y), \\
F_{3}(x, y)=\sqrt{x y} J_{\nu}(x) J_{\nu}^{\prime}(y), & F_{4}(x, y)=\sqrt{x y} J_{\nu}^{\prime}(x) J_{\nu}^{\prime}(y) .
\end{array}
$$

Then we have

$$
\begin{aligned}
& K_{\nu}^{1}(x, y) \\
& \quad=\frac{2}{u^{2}}\left(\left(2 \nu^{2}-v\right) F_{1}(x, y)-2 x \frac{v}{u} F_{2}(x, y)+2 y \frac{v}{u} F_{3}(x, y)-2 x y F_{4}(x, y)\right)
\end{aligned}
$$

whenever $x \neq y$. This may be checked by noting that

$$
K_{\nu}^{1}(x, y)=2 \mathcal{D}^{2} F_{1}(x, y)
$$

where

$$
\mathcal{D}=\frac{1}{u}\left(y \frac{\partial}{\partial y}-x \frac{\partial}{\partial x}\right)
$$

using $\mathcal{D}(f g h)=\mathcal{D}(f) g h+f \mathcal{D}(g) h+f g \mathcal{D}(h)$ and $\mathcal{D}\left(x^{a} y^{b} u^{-c}\right)=x^{a} y^{b} u^{-c-2}((b-$ $a) u+2 c v), a, b, c \in \mathbb{R}$, and observing how $\mathcal{D}$ acts on $F_{i}, i=1,2,3$ :

$$
\begin{aligned}
& \mathcal{D}\left(F_{1}\right)=\frac{1}{u}\left(-x F_{2}+y F_{3}\right), \\
& \mathcal{D}\left(F_{2}\right)=\frac{1}{u}\left(-\frac{\nu^{2}-x^{2}}{x} F_{1}+F_{2}+y F_{4}\right), \\
& \mathcal{D}\left(F_{3}\right)=\frac{1}{u}\left(\frac{\nu^{2}-y^{2}}{y} F_{1}-F_{3}-x F_{4}\right) ;
\end{aligned}
$$

the identities are obtained by using the fact that $J_{\nu}(x)$ satisfies Bessel's differential equation $J_{\nu}^{\prime \prime}(x)=\left(\left(\nu^{2}-x^{2}\right) / x^{2}\right) J_{\nu}(x)-(1 / x) J_{\nu}^{\prime}(x)$. To verify (14) we first use Lommel's formula

$$
\int_{0}^{s} t J_{\nu}(x t) J_{\nu}(y t) d t=s \frac{x J_{\nu+1}(s x) J_{\nu}(s y)-y J_{\nu}(s x) J_{\nu+1}(s y)}{x^{2}-y^{2}},
$$

with $s=1$, the identity $x J_{\nu+1}(x)=\nu J_{\nu}(x)-x J_{\nu}^{\prime}(x)$ and the expression on $\mathcal{D}\left(F_{1}\right)$ to obtain $K_{\nu}^{0}(x, y)=\mathcal{D} F_{1}(x, y)$, where $K_{\nu}^{0}(x, y)$ denotes the integral kernel of $S_{\nu}^{0}=$ $S_{\nu, 1}^{0}$. Then, integrating by parts, using Lommel's formula and the expression on 
$\mathcal{D}\left(F_{1}\right)$ shows that

$$
\begin{aligned}
K_{\nu}^{1}(x, y) & =\sqrt{x y} \int_{0}^{1}\left(1-s^{2}\right) s J_{\nu}(x s) J_{\nu}(y s) d s \\
& =2 \sqrt{x y} \int_{0}^{1} s \cdot s \frac{x J_{\nu+1}(s x) J_{\nu}(s y)-y J_{\nu}(s x) J_{\nu+1}(s y)}{x^{2}-y^{2}} d s \\
& =2 \sqrt{x y} \int_{0}^{1} s \mathcal{D}\left(J_{\nu}(s x) J_{\nu}(s y)\right) d s \\
& =2 \mathcal{D} K_{\nu}^{0}(x, y) .
\end{aligned}
$$

This completes checking (14). We should add at this point that according to the Laguerre case, (14) could be called a Campbell-type formula.

If $\nu=-1 / 2$ then $J_{-1 / 2}(u)=(2 /(\pi u))^{1 / 2} \cos u$ and $\mathcal{H}_{-1 / 2}$ reduces to the cosine transform. A direct calculation of the integral representing $K_{-1 / 2}^{1}(x, y)$ then shows that

$$
\begin{aligned}
K_{-1 / 2}^{1}(x, y)=\frac{2}{\pi}\left(\frac{1}{(x-y)^{3}} \sin (x-y)\right. & -\frac{1}{(x-y)^{2}} \cos (x-y)+\frac{1}{(x+y)^{3}} \sin (x+y) \\
& \left.-\frac{1}{(x+y)^{2}} \cos (x+y)\right)
\end{aligned}
$$

Similarly, if $\nu=1 / 2$ then $J_{1 / 2}(u)=(2 /(\pi u))^{1 / 2} \sin u, \mathcal{H}_{1 / 2}$ reduces to the sine transform and

$$
\begin{aligned}
K_{1 / 2}^{1}(x, y)=\frac{2}{\pi}\left(\frac{1}{(x-y)^{3}} \sin (x-y)\right. & -\frac{1}{(x-y)^{2}} \cos (x-y)-\frac{1}{(x+y)^{3}} \sin (x+y) \\
& \left.+\frac{1}{(x+y)^{2}} \cos (x+y)\right)
\end{aligned}
$$

These two exact formulas (consistent with (13)!) give an immediate insight on how estimates of $K_{\nu}^{1}(x, y)$ should look like, for a single $\nu$, in different regions of the quarter plane. For estimates uniform on $\nu$, (13) together with the uniform estimates that follow are required.

We will make an extensive use of the following uniform pointwise estimates of Bessel functions and their first derivatives: with a constant $D$, independent of $\nu$ and $x$, for $\nu \geq 1$,

$$
\left|x^{1 / 2} J_{\nu}(x)\right| \leq D \begin{cases}x^{\nu+1 / 2}\left(\Gamma(\nu+1) 2^{\nu}\right)^{-1}, & 0<x<\nu / 2 \\ \nu^{1 / 4}\left(\nu^{1 / 3}+|x-\nu|\right)^{-1 / 4}, & \nu / 2<x<2 \nu \\ 1, & 2 \nu<x<\infty\end{cases}
$$

and

$$
\left|x^{1 / 2} J_{\nu}^{\prime}(x)\right| \leq D \begin{cases}\nu x^{\nu-1 / 2}\left(\Gamma(\nu+1) 2^{\nu}\right)^{-1}, & 0<x<\nu / 2, \\ \nu^{-1 / 4}\left(\nu^{1 / 3}+|x-\nu|\right)^{1 / 4}, & \nu / 2<x<2 \nu \\ 1, & 2 \nu<x<\infty\end{cases}
$$

and, for $-1<\nu<1$,

$$
\left|x^{1 / 2} J_{\nu}(x)\right| \leq D \begin{cases}x^{\nu+1 / 2}(\Gamma(\nu+1))^{-1}, & 0<x<1 / 2 \\ 1, & 1 / 2<x<\infty\end{cases}
$$

and

$$
\left|x^{1 / 2} J_{\nu}^{\prime}(x)\right| \leq D \begin{cases}x^{\nu-1 / 2}(\Gamma(\nu+1))^{-1}, & 0<x<1 / 2 \\ 1, & 1 / 2<x<\infty\end{cases}
$$


In the case $\nu=0$, the bound $x^{-1 / 2}$ in (18) has to be replaced by $x^{3 / 2}$. (15) and (16) are direct consequences of the delicate bounds done by Barceló and Córdoba; they follow from the table on p. 661 of [1], or p. 24 of [2]. The transition point $x=\nu / 2$ in (15) and (16) may be replaced (clearly, with a different $D)$ by $(1+\varepsilon) \nu / 2$, or in (17) and (18), $x=1 / 2$ may be replaced by $(1+\varepsilon) / 2$, where $0<\varepsilon<1$ is chosen earlier; cf. [7] for additional comments.

Denoting by $\Phi_{\nu}(x)$ the function that appears on the right of (15) when $\nu \geq 1$ or on the right of $(17)$ when $-1<\nu<1$, we have

$$
\left|\sqrt{x} J_{\nu}(x)\right| \leq D \Phi_{\nu}(x) .
$$

Accordingly, (16) and (18) may be written in the following form

$$
\left|\sqrt{x} J_{\nu}^{\prime}(x)\right| \leq C \frac{\sqrt{\left|\nu^{2}-x^{2}\right|+x^{4 / 3}}}{x} \Phi_{\nu}(x)
$$

when $\nu \geq 1$ and

$$
\left|\sqrt{x} J_{\nu}^{\prime}(x)\right| \leq C \frac{x+1}{x} \Phi_{\nu}(x)
$$

when $-1<\nu<1$ with the exception for $\nu=0$; then $(x+1) / x$ is replaced by $x /(x+1)$.

For the purpose of kernel estimates that are proved in Lemma 1 it is much more convenient to split $(\nu / 2,2 \nu)$ into the three intervals $\left(\nu / 2, \nu-\nu^{1 / 3}\right),\left(\nu-\nu^{1 / 3}, \nu+\nu^{1 / 3}\right)$ and $\left(\nu+\nu^{1 / 3}, 2 \nu\right)$ and, instead of $\Phi_{\nu}(x)$ in (19) use, if $\nu \geq 3$, say, the (equivalent) bound by

$$
\widetilde{\Phi}_{\nu}(x)= \begin{cases}x^{\nu+1 / 2}\left(\Gamma(\nu+1) 2^{\nu}\right)^{-1}, & 0<x \leq \nu / 2, \\ \nu^{1 / 4}(\nu-x)^{-1 / 4}, & \nu / 2<x \leq \nu-\nu^{1 / 3}, \\ \nu^{1 / 6}, & \nu-\nu^{1 / 3}<x \leq \nu+\nu^{1 / 3}, \\ \nu^{1 / 4}(x-\nu)^{-1 / 4}, & \nu+\nu^{1 / 3}<x \leq 2 \nu, \\ 1, & 2 \nu<x .\end{cases}
$$

We have, for certain $a_{\nu}, b_{\nu}$ and $c_{\nu}$, the asymptotic

$$
\sqrt{t} J_{\nu}(t)=\sqrt{2 / \pi}\left(\cos \left(t+a_{\nu}\right)+b_{\nu} \frac{\sin \left(t+c_{\nu}\right)}{t}+O\left(t^{-2}\right)\right), \quad t \rightarrow \infty .
$$

At several places of the next section, without further refering to it, Stirling's formula is used:

$$
\lim _{\nu \rightarrow \infty} \nu^{\nu+1 / 2} e^{-\nu} \Gamma(\nu+1)^{-1}=(2 \pi)^{-1 / 2} .
$$

Given $p, 1 \leq p \leq \infty, p^{\prime}$ denotes its conjugate, $1 / p+1 / p^{\prime}=1$.

\section{KERNEL ESTIMATES AND BOUNDS FOR $K_{\nu}^{1}$ IN DIFFERENT REGIONS}

In the sequel we write $\bar{\nu}=\nu$ if $\nu \geq 1$ and $\bar{\nu}=1$ if $-1<\nu \leq 1$. Also, we use the notation

$$
w_{a, b}(x)=x^{a}(1+x)^{b-a} .
$$

The lemmas that follow give proper estimates of the kernel $K_{\nu}^{1}(x, y)$ in different regions of the quarter plane $(0, \infty) \times(0, \infty)$. The corresponding propositions furnish weighted $L^{p}$ bounds of associated kernel operators restricted to relevant regions with minimal assumptions required on $a, b, A, B$. In the propositions the constant $C$ will depend on $\alpha, p, a, b, A, B$ but will not depend on $\nu \geq \alpha$ and $f$. At several places the usual interpretation of the $L^{p}$ norm is needed when $p=\infty$. 
Lemma 1. There exists $C>0$ independent of $\nu>-1$ such that

$$
\left|K_{\nu}^{1}(x, y)\right| \leq C \begin{cases}(x y)^{\nu+1 / 2} /\left(\Gamma(\nu+2) 2^{\nu}\right)^{2}, & 0<x, y<2 \bar{\nu} / 3 \\ 1, & x, y>\bar{\nu} / 2 .\end{cases}
$$

Proof. Using (15) and (17) gives, for $0<x, y<2 \bar{\nu} / 3$,

$$
\left|K_{\nu}^{1}(x, y)\right| \leq C \frac{(x y)^{\nu+1 / 2}}{\Gamma(\nu+1)^{2} 2^{2 \nu}} \int_{0}^{1}\left(1-s^{2}\right) s^{2 \nu+1} d s .
$$

To get the required bound we use

$$
\int_{0}^{1}\left(1-s^{2}\right) s^{2 \nu+1} d s=\frac{1}{2} \frac{\Gamma(\nu+1) \Gamma(2)}{\Gamma(\nu+3)}
$$

and then majorize the last expression by $C(1+\nu)^{-2}$. To prove the estimate for $x, y>\bar{\nu} / 2$ we note that by Schwarz's inequality we have $\left|K_{\nu}^{1}(x, y)\right|^{2} \leq A_{\nu}(x) A_{\nu}(y)$, where

$$
A_{\nu}(x)=\int_{0}^{1}\left(1-s^{2}\right)\left(\sqrt{x s} J_{\nu}(x s)\right)^{2} d s .
$$

It is therefore sufficient to check that $A_{\nu}(x) \leq C$ for $x>\bar{\nu} / 2$. In what follows we consider the case $\nu \geq 1$ only (analysing the case $-1<\nu<1$ is much easier). In fact we also assume that $\nu \geq 3$. We consider four cases to estimate $A_{\nu}(x)$ : $\nu / 2<x \leq \nu-\nu^{1 / 3}, \nu-\nu^{1 / 3}<x \leq \nu+\nu^{1 / 3}, \nu+\nu^{1 / 3}<x \leq 2 \nu$, and $2 \nu<x$.

Case 1: $\nu / 2<x \leq \nu-\nu^{1 / 3}$. By (19), (here and in the sequel we use (19) with $\widetilde{\Phi}_{\nu}(x)$ in place of $\left.\Phi_{\nu}(x)\right)$

$$
\left|\sqrt{x s} J_{\nu}(x s)\right| \leq C \begin{cases}(x s)^{\nu+1 / 2}\left(\Gamma(\nu+1) 2^{\nu}\right)^{-1}, & 0<s \leq \nu /(2 x) \\ \nu^{1 / 4}(\nu-x s)^{-1 / 4}, & \nu /(2 x)<s<1\end{cases}
$$

Therefore,

$$
\begin{aligned}
A_{\nu}(x) \leq C\left(x^{2 \nu+1}\left(\Gamma(\nu+1) 2^{\nu}\right)^{-2}\right. & \int_{0}^{\nu /(2 x)}\left(1-s^{2}\right) s^{2 \nu+1} d s \\
& \left.+\nu^{1 / 2} \int_{\nu /(2 x)}^{1}\left(1-s^{2}\right)(\nu-x s)^{-1 / 2} d s\right) .
\end{aligned}
$$

The first summand above is bounded by using

$$
\int_{0}^{\nu /(2 x)}\left(1-s^{2}\right) s^{2 \nu+1} d s \leq C \frac{1}{2(\nu+1)}\left(\frac{\nu}{2 x}\right)^{2(\nu+1)}
$$

and Stirling's formula. For the second summand we write

$$
\int_{\nu /(2 x)}^{1}\left(1-s^{2}\right)(\nu-x s)^{-1 / 2} d s \leq \int_{\nu /(2 x)}^{\nu / x}(\nu-x s)^{-1 / 2} d s
$$

and note that the last integral is bounded by $C \nu^{-1 / 2}$ which gives the correct bound of the second summand. The claim, $A_{\nu}(x) \leq C$, now follows.

Case 2: $\nu-\nu^{1 / 3}<x<\nu+\nu^{1 / 3}$. From (19), the following estimate holds:

$$
\left|\sqrt{x s} J_{\nu}(x s)\right| \leq C \begin{cases}(x s)^{\nu+1 / 2}\left(\Gamma(\nu+1) 2^{\nu}\right)^{-1}, & 0<s \leq \nu /(2 x) \\ \nu^{1 / 4}(\nu-x s)^{-1 / 4}, & \nu /(2 x)<s \leq\left(\nu-\nu^{1 / 3}\right) / x \\ \nu^{1 / 6}, & \left(\nu-\nu^{1 / 3}\right) / x<s<1\end{cases}
$$


Therefore,

$$
\begin{aligned}
& A_{\nu}(x) \leq C\left(x^{2 \nu+1}\left(\Gamma(\nu+1) 2^{\nu}\right)^{-2} \int_{0}^{\nu /(2 x)}\left(1-s^{2}\right) s^{2 \nu+1} d s\right. \\
& \left.+\nu^{1 / 2} \int_{\nu /(2 x)}^{\left(\nu-\nu^{1 / 3}\right) / x}\left(1-s^{2}\right)(\nu-x s)^{-1 / 2} d s+\nu^{1 / 3} \int_{\left(\nu-\nu^{1 / 3}\right) / x}^{1}\left(1-s^{2}\right) d s\right) .
\end{aligned}
$$

The first summand is that from Case 1 . The same is with the second summand once we enlarge the upper integral limit from $\left(\nu-\nu^{1 / 3}\right) / x$ to 1 . The integral contained in the third summand is easily seen to be bounded by $C \nu^{-1 / 3}$ which gives the correct bound of the summand. The claim, $A_{\nu}(x) \leq C$, again follows.

Case 3: $\nu+\nu^{1 / 3}<x \leq 2 \nu$. As in the previous cases we use (19) obtaining:

$$
\left|\sqrt{x s} J_{\nu}(x s)\right| \leq C \begin{cases}(x s)^{\nu+1 / 2}\left(\Gamma(\nu+1) 2^{\nu}\right)^{-1}, & 0<s \leq \nu /(2 x), \\ \nu^{1 / 4}(\nu-x s)^{-1 / 4}, & \nu /(2 x)<s \leq\left(\nu-\nu^{1 / 3}\right) / x, \\ \nu^{1 / 6}, & \left(\nu-\nu^{1 / 3}\right) / x<s \leq\left(\nu+\nu^{1 / 3}\right) / x \\ \nu^{1 / 4}(x s-\nu)^{-1 / 4}, & \left(\nu+\nu^{1 / 3}\right) / x<s<1 .\end{cases}
$$

Next, we bound $A_{\nu}(x)$ by splitting the integration accordingly. The first two resulting terms are exactly those from Case 2. The same is with the third one once the upper integral limit is enlarged from $\left(\nu+\nu^{1 / 3}\right) / x$ to 1 . The fourth resulting term is

$$
\nu^{1 / 2} \int_{\left(\nu+\nu^{1 / 3}\right) / x}^{1}\left(1-s^{2}\right)(x s-\nu)^{-1 / 2} d s
$$

and the required claim follows since

$$
\int_{\left(\nu+\nu^{1 / 3}\right) / x}^{1}\left(1-s^{2}\right)(x s-\nu)^{-1 / 2} d s \leq C x^{-1 / 2} \int_{\nu / x}^{1}\left(s-\frac{\nu}{x}\right)^{-1 / 2} d s \leq C \nu^{-1 / 2} .
$$

Case $4: 2 \nu<x$. In this situation we have

$$
\left|\sqrt{x s} J_{\nu}(x s)\right| \leq C \begin{cases}(x s)^{\nu+1 / 2}\left(\Gamma(\nu+1) 2^{\nu}\right)^{-1}, & 0<s \leq \nu /(2 x), \\ \nu^{1 / 4}(\nu-x s)^{-1 / 4}, & \nu /(2 x)<s \leq\left(\nu-\nu^{1 / 3}\right) / x \\ \nu^{1 / 6}, & \left(\nu-\nu^{1 / 3}\right) / x<s \leq\left(\nu+\nu^{1 / 3}\right) / x \\ \nu^{1 / 4}(x s-\nu)^{-1 / 4}, & \left(\nu+\nu^{1 / 3}\right) / x<s \leq 2 \nu / x \\ 1, & 2 \nu / x<s<1 .\end{cases}
$$

Again, we bound $A_{\nu}(x)$ by properly splitting the integration and noting that the first three resulting terms are those from Case 3. The same is with the fourth one after replacing the upper integral limit $2 \nu / x$ by 1 . The fifth resulting term is $\int_{2 \nu / x}^{1}\left(1-s^{2}\right) d s$ and it is obviously bounded by a constant. This finishes the proof of Case 4 hence the lemma.

Proposition 1. Let $\alpha>-1$ and $1 \leq p \leq \infty$. Assuming $a>-1 / p-(\alpha+1 / 2)$ ( $\geq$ if $p=\infty)$ and $A<1-1 / p+(\alpha+1 / 2)(\leq$ if $p=1)$ we have

$$
\int_{0}^{2 \bar{\nu} / 3}\left(w_{a, b}(x) \int_{0}^{2 \bar{\nu} / 3}\left|K_{\nu}^{1}(x, y) f(y)\right| d y\right)^{p} d x \leq C \int_{0}^{2 \bar{\nu} / 3}\left|f(y) w_{A, B}(y)\right|^{p} d y
$$

for $\nu \geq \alpha$, while, assuming in addition $b \leq B$, for $\nu \geq \alpha$ we have

$$
\int_{\bar{\nu} / 2}^{\infty}\left(w_{a, b}(x) \int_{\bar{\nu} / 2}^{\infty} \chi_{D}(x, y)\left|K_{\nu}^{1}(x, y) f(y)\right| d y\right)^{p} d x \leq C \int_{\bar{\nu} / 2}^{\infty}\left|f(y) w_{A, B}(y)\right|^{p} d y
$$

where $D=\{(x, y):|x-y| \leq 1\}$. 
Proof. Proving (23) use the bound on $\left|K_{\nu}^{1}(x, y)\right|$ from Lemma 1 and consider $p=1$ first. Then split the integration regions $[0,2 \bar{\nu} / 3]$ in the double integral using $2 / 3$ as breakpoints and change the order of integration to end up with four integrals to be estimated by a constant multiple of either $\int_{0}^{2 / 3}|f(y)| y^{A} d y$ or $\int_{2 / 3}^{2 \bar{\nu} / 3}|f(y)| y^{B} d y$. If the $y$-region of integration is $[0,2 / 3]$, then merely replace $y^{\nu+1 / 2}$ by $y^{A}$ (using the assumption $A \leq \alpha+1 / 2$ ) and note that the integrals

$$
\int_{0}^{2 / 3} x^{a+\nu+1 / 2} d x, \quad \int_{2 / 3}^{2 \bar{\nu} / 3} x^{b+\nu+1 / 2} d x,
$$

when multiplied by $\left(\Gamma(\nu+2) 2^{\nu}\right)^{-2}$ give bounded functions of $\nu \geq \alpha$. If the $y$ region of integration is $[2 / 3,2 \bar{\nu} / 3]$ then, for large values of $\nu$ say, replace $y^{\nu+1 / 2}$ by $(2 \nu / 3)^{\nu+1 / 2-B} y^{B}$ and, in the case of the $x$-integration over $[2 / 3,2 \nu / 3]$, note that

$$
\frac{1}{\left(\Gamma(\nu+2) 2^{\nu}\right)^{2}}\left(\frac{2}{3} \nu\right)^{\nu+1 / 2-B} \int_{2 / 3}^{2 \nu / 3} x^{b+\nu+1 / 2} d x
$$

is a bounded function of large $\nu$ (note that the possible growth of $x^{b-B-2}$ is compensated by the decay of $\left.(e / 3)^{\nu}\right)$. The remaining case of the $x$-integration over $[0,2 / 3]$ is similar (and easier). In the case $1<p<\infty$ apply the analogous argument using Hölder's inequality in appropriate places (cf. also the proof of Lemma 2.1 in [7]). Consider now $p=\infty$. It is then sufficient to show that, for $\nu \geq \alpha$, the quantities

$$
\frac{1}{\left(\Gamma(\nu+2) 2^{\nu}\right)^{2}} \sup _{0<x<2 / 3}\left[x^{a+\nu+1 / 2} \int_{0}^{2 \bar{\nu} / 3} y^{\nu+1 / 2}|f(y)| d y\right]
$$

and

$$
\frac{1}{\left(\Gamma(\nu+2) 2^{\nu}\right)^{2}} \sup _{2 / 3<x<2 \bar{\nu} / 3}\left[x^{b+\nu+1 / 2} \int_{0}^{2 \bar{\nu} / 3} y^{\nu+1 / 2}|f(y)| d y\right]
$$

are bounded by a constant (independent of $\nu \geq \alpha$ ) multiplied by the sum

$$
\sup _{0<y<2 / 3} y^{A}|f(y)|+\sup _{2 / 3<y<2 \bar{\nu} / 3} y^{B}|f(y)| \text {. }
$$

This is easily done by using the assumptions $a \geq-(\alpha+1 / 2)$ and $A<1+(\alpha+1 / 2)$.

Proving (24) use Lemma 1 and note that only $\nu \geq 1$ may be considered. Let $p=1$. It is sufficient to show that

$$
\int_{\nu / 2}^{\infty} x^{b} \int_{\nu / 2}^{\infty} \chi_{[0,1]}(|x-y|)|f(y)| d y d x \leq C \int_{\nu / 2}^{\infty}|f(y)| y^{B} d y
$$

with $C$ independent of $\nu \geq 1$. The inequality immediately follows by changing the order of integration, noting that for $y \geq \nu / 2$ and $\nu \geq 1$

$$
\int_{\nu / 2}^{\infty} x^{b} \chi_{[0,1]}(|x-y|) d x \leq C y^{b}
$$

and using $b \leq B$. Consider now the case $1<p \leq \infty$. By using $b \leq B$ it is easily seen that (24) is implied by

$$
\int_{\nu / 2}^{\infty}\left(\int_{x-1}^{x+1}\left|\chi_{[\nu / 2, \infty]}(y) g(y)\right| d y\right)^{p} d x \leq C \int_{\nu / 2}^{\infty}|g(y)|^{p} d y
$$

where $\nu \geq 1$. This, in order, follows from the well-known standard norm inequality for the maximal function since, for $x \geq \nu / 2$ and $\nu \geq 1$,

$$
\int_{x-1}^{x+1}\left|\chi_{[\nu / 2, \infty]}(y) g(y)\right| d y \leq C M\left(\chi_{[\nu / 2, \infty]} g\right)(x) .
$$


Lemma 2. Let $\alpha>-1$. There exists a constant $C$ independent of $\nu \geq \alpha$ such that for $|x-y| \geq 1$ and $x, y \geq 5 \bar{\nu} / 4$,

$$
\left|K_{\nu}^{1}(x, y)\right| \leq C|x-y|^{-2} .
$$

Proof. Using (13) gives

$$
\left|K_{\nu}^{1}(x, y)\right| \leq 2|u|^{-2}\left(\left|2 \nu^{2}-v\right|\left|F_{1}\right|+\frac{2 v}{|u|}\left(x\left|F_{2}\right|+y\left|F_{3}\right|\right)+2 x y\left|F_{4}\right|\right) .
$$

Since $\left|F_{i}\right| \leq C$ on $x, y \geq \bar{\nu}$, see (15)-(18), the conclusion then follows by noting that each of the terms, $\left|2 \nu^{2}-\left(x^{2}+y^{2}\right)\right|, v, x y$, when divided by $(x+y)^{2}$ is bounded on the indicated range of $x$ and $y$.

Proposition 2. Let $\alpha>-1,1 \leq p \leq \infty, b \leq B, b<2-1 / p(\leq$ if $p=\infty)$, and $B>-1-1 / p(\geq$ if $p=1)$. Then, for $\nu \geq \alpha$,

$$
\int_{5 \bar{\nu} / 4}^{\infty}\left(w_{a, b}(x) \int_{5 \bar{\nu} / 4}^{\infty} \chi_{D^{c}}(x, y)\left|K_{\nu}^{1}(x, y) f(y)\right| d y\right)^{p} d x \leq C \int_{5 \bar{\nu} / 4}^{\infty}\left|f(y) w_{A, B}(y)\right|^{p} d y,
$$

where $D^{c}=\{(x, y):|x-y| \geq 1\}$.

Proof. Consider first $p=1$. Then (27) reduces to showing that

$$
\int_{5 \bar{\nu} / 4}^{\infty} \int_{5 \bar{\nu} / 4}^{\infty} \chi_{[1, \infty)}(|x-y|) x^{b} y^{-B} \frac{|g(y)|}{|x-y|^{2}} d y d x \leq C \int_{2 \bar{\nu}}^{\infty}|g(y)| d y
$$

and this is a consequence of (this time $b<1$ is used)

$$
\sup _{\nu \geq \alpha} \sup _{y \geq 5 \bar{\nu} / 4}\left[y^{-B} \int_{5 \bar{\nu} / 4}^{\infty} x^{b} \chi_{[1, \infty)}(|x-y|) \frac{1}{|x-y|^{2}} d x\right]<\infty .
$$

In the case $p=\infty,(27)$ reduces to showing that

$$
\sup _{x \geq 5 \bar{\nu} / 4}\left[x^{b} \int_{5 \bar{\nu} / 4}^{\infty} \chi_{D^{c}}(x, y) \frac{|g(y)|}{|x-y|^{2}} y^{-B} d y\right] \leq C \sup _{y \geq 5 \bar{\nu} / 4}|g(y)|
$$

and this easily follows by noting that $(B>-1$ is used)

$$
\sup _{\bar{\nu} \geq \alpha} \sup _{x \geq 5 \bar{\nu} / 4}\left[x^{b} \int_{2 \bar{\nu}}^{\infty} \chi_{[1, \infty)}(|x-y|) \frac{1}{y^{B}|x-y|^{2}} d y\right]<\infty .
$$

Consider now the case $1<p<\infty$. We decompose the $y$-range in $[5 \bar{\nu} / 4, x / 2]$, $[x / 2,2 x]$ and $[2 x, \infty)$. Then,

$$
\begin{aligned}
& \int_{5 \bar{\nu} / 4}^{\infty}\left(w_{a, b}(x) \int_{5 \bar{\nu} / 4}^{x / 2} \chi_{D^{c}}(x, y) \mid\right.\left.K_{\nu}^{1}(x, y) f(y) \mid d y\right)^{p} d x \\
& \leq C \int_{5 \bar{\nu} / 4}^{\infty}\left(x^{b-2} \int_{5 \bar{\nu} / 4}^{x}|f(y)| d y\right)^{p} d x
\end{aligned}
$$

and an application of Hölder's inequality shows that the last expression is bounded by the right side of (27). Similarly,

$\int_{5 \bar{\nu} / 4}^{\infty}\left(w_{a, b}(x) \int_{2 x}^{\infty} \chi_{D^{c}}(x, y)\left|K_{\nu}^{1}(x, y) f(y)\right| d y\right)^{p} d x \leq C \int_{5 \bar{\nu} / 4}^{\infty}\left(x^{b} \int_{x}^{\infty} \frac{|f(y)|}{y^{2}} d y\right)^{p} d x$ and again Hölder's inequality does the job. Proving

$$
\int_{5 \bar{\nu} / 4}^{\infty}\left(w_{a, b}(x) \int_{x / 2}^{2 x} \chi_{D^{c}}(x, y)\left|K_{\nu}^{1}(x, y) f(y)\right| d y\right)^{p} d x \leq C \int_{5 \bar{\nu} / 4}^{\infty}\left|w_{A, B}(x) f(x)\right|^{p} d x
$$


reduces to showing that

$$
\int_{5 \bar{\nu} / 4}^{\infty} x^{p(b-B)}\left(\int_{x / 2}^{2 x} \chi_{[1, \infty]}(|x-y|) \frac{|g(y)|}{|x-y|^{2}} d y\right)^{p} d x \leq C \int_{5 \bar{\nu} / 4}^{\infty}|g(x)|^{p} d x .
$$

By the assumption $b \leq B$, we obtain

$$
\begin{aligned}
& \int_{5 \bar{\nu} / 4}^{\infty} x^{p(b-B)}\left(\int_{x / 2}^{2 x} \chi_{[1, \infty]}(|x-y|) \frac{|g(y)|}{|x-y|^{2}} d y\right)^{p} d x \\
\leq & C \int_{5 \bar{\nu} / 4}^{\infty}\left(\sum_{k=1}^{\infty} \int_{|x-y| \sim 2^{k}} \frac{|g(y)|}{|x-y|^{2}} \chi_{[5 \bar{\nu} / 4, \infty]}(y) d y\right)^{p} d x \\
\leq & C \int_{5 \bar{\nu} / 4}^{\infty}\left(\sum_{k=1}^{\infty} 2^{-2 k} \int_{|x-y| \sim 2^{k}}|g(y)| \chi_{[5 \bar{\nu} / 4, \infty]}(y) d y\right)^{p} d x \\
\leq & C \int_{5 \bar{\nu} / 4}^{\infty} M\left(\chi_{[5 \bar{\nu} / 4, \infty)} g\right)(x)^{p} d x .
\end{aligned}
$$

Using the fact that $M$ is a bounded operator on $L^{p}$ for $1<p<\infty$, we conclude the proof of Proposition 2.

Lemma 3. There exists a constant $C$ independent of $\nu \geq 1$ such that for $|x-y| \geq 1$ and $\nu / 2 \leq x, y \leq 3 \nu / 2$,

(28) $\left|K_{\nu}^{1}(x, y)\right| \leq C|x-y|^{-2}\left(\left(\frac{|\nu-x|+\nu^{1 / 3}}{|\nu-y|+\nu^{1 / 3}}\right)^{1 / 4}+\left(\frac{|\nu-y|+\nu^{1 / 3}}{|\nu-x|+\nu^{1 / 3}}\right)^{1 / 4}\right)$.

Proof. Applying (19) and (20) to (26) leads to

$$
\begin{aligned}
& \left|K_{\nu}^{1}(x, y)\right| \leq C \Phi_{\nu}(x) \Phi_{\nu}(y)|u|^{-2}\left(\left|2 \nu^{2}-v\right|+\frac{v}{|u|}\left(\left|\nu^{2}-x^{2}\right|+x^{4 / 3}\right)^{1 / 2}\right. \\
& \left.\quad+\frac{v}{|u|}\left(\left|\nu^{2}-y^{2}\right|+y^{4 / 3}\right)^{1 / 2}+\left(\left|\nu^{2}-x^{2}\right|+x^{4 / 3}\right)^{1 / 2}\left(\left|\nu^{2}-y^{2}\right|+y^{4 / 3}\right)^{1 / 2}\right) .
\end{aligned}
$$

Then the estimates for $\Phi_{\nu}$ on the range $(\nu / 2,3 \nu / 2)$ give

$$
\begin{aligned}
& \left|K_{\nu}^{1}(x, y)\right| \leq C|x-y|^{-2}\left(|\nu-x|+\nu^{1 / 3}\right)^{-1 / 4}\left(|\nu-y|+\nu^{1 / 3}\right)^{-1 / 4} \nu^{-3 / 2} \\
& \times\left(\nu[|\nu-x|+|\nu-y|]+\nu^{3 / 2}\left[\left(|\nu-x|+\nu^{1 / 3}\right)^{1 / 2}+\left(|\nu-y|+\nu^{1 / 3}\right)^{1 / 2}\right]\right. \\
& \left.+\nu\left(|\nu-x|+\nu^{1 / 3}\right)^{1 / 2}\left(|\nu-y|+\nu^{1 / 3}\right)^{1 / 2}\right) .
\end{aligned}
$$

A careful analysis then shows that each of the three resulting summands is bounded by the right side of (28).

Proposition 3. Let $\alpha \geq 1,1 \leq p \leq \infty$, and $b \leq B$. Then, for $\nu \geq \alpha$,

$$
\int_{\nu / 2}^{3 \nu / 2}\left(w_{a, b}(x) \int_{\nu / 2}^{3 \nu / 2} \chi_{D^{c}}(x, y)\left|K_{\nu}^{1}(x, y) f(y)\right| d y\right)^{p} d x \leq C \int_{\nu / 2}^{3 \nu / 2}\left|f(y) w_{A, B}(y)\right|^{p} d y .
$$

Proof. By using (28) and the assumption $b \leq B$, (30) will follow from

$$
\begin{gathered}
\left(\int_{\nu / 2}^{3 \nu / 2}\left(\int_{\nu / 2}^{3 \nu / 2} \chi_{D^{c}}(x, y) \frac{|f(y)|}{|x-y|^{2}}\left(\frac{|\nu-x|+\nu^{1 / 3}}{|\nu-y|+\nu^{1 / 3}}\right)^{1 / 4} d y\right)^{p} d x\right)^{1 / p} \\
\leq C\left(\int_{\nu / 2}^{3 \nu / 2}|f(y)|^{p} d y\right)^{1 / p}
\end{gathered}
$$


and an analogous inequality with the second term from the right side of (28) involved. Since in the latter case the argument is completely similar to what we present below we do not repeat it. The change of variables $X=\nu^{-1 / 3}(x-\nu)$, $Y=\nu^{-1 / 3}(y-\nu)$, shows that the left side of (31) equals $\nu^{-1 / 3+1 /(3 p)}$ multiplied by

$$
\left(\int_{-\nu^{2 / 3} / 2}^{\nu^{2 / 3} / 2}\left(\int_{-\nu^{2 / 3} / 2}^{\nu^{2 / 3} / 2} \chi_{\left\{|X-Y|>\nu^{-1 / 3}\right\}}(X, Y) \frac{|F(Y)|}{|X-Y|^{2}}\left(\frac{1+|X|}{1+|Y|}\right)^{1 / 4} d Y\right)^{p} d X\right)^{1 / p},
$$

with $F(Y)=f\left(Y \nu^{1 / 3}+\nu\right)$. By symmetry, it is now enough to majorize the quantity

$$
\left(\int_{0}^{\nu^{2 / 3} / 2}\left(\int_{-\nu^{2 / 3} / 2}^{\nu^{2 / 3} / 2} \chi_{\left\{|X-Y|>\nu^{-1 / 3}\right\}}(X, Y) \frac{|F(Y)|}{|X-Y|^{2}}\left(\frac{1+|X|}{1+|Y|}\right)^{1 / 4} d Y\right)^{p} d X\right)^{1 / p}
$$

by $\nu^{1 / 3-1 /(3 p)}$ times the right side of (31). We decompose the $Y$-range into five intervals $\left[-\nu^{2 / 3} / 2,-X\right],[-X, 0],[0, X / 2],[X / 2,2 X]$ and $\left[2 X, \nu^{2 / 3} / 2\right]$ (the extreme intervals may be void, the second one may reduce to $\left[-\nu^{2 / 3} / 2,0\right]$ ) and denote the resulting double integrals by $I_{i}, i=1, \ldots, 5$. We will focus on proving that

$$
I_{i} \leq C \nu^{1 / 3-1 /(3 p)}\left(\int_{\nu / 2}^{3 \nu / 2}|f(y)|^{p} d y\right)^{1 / p}, \quad i=1, \ldots, 5 .
$$

Let $i=1$. If $Y \in\left[-\nu^{2 / 3} / 2,-X\right]$ then $|X-Y|^{-2} \leq C|Y|^{-2}$, the change of variable $Y=-T$ and Hölder's inequality produce the following bound of $I_{1}$ :

$$
\begin{aligned}
&\left(\int_{0}^{\nu^{2 / 3} / 2}\left(\int_{-\nu^{2 / 3} / 2}^{-X} \chi_{\left\{|X-Y|>\nu^{-1 / 3}\right\}}(X, Y) \frac{|F(Y)|}{|X-Y|^{2}}\left(\frac{1+|X|}{1+|Y|}\right)^{1 / 4} d Y\right)^{p} d X\right)^{1 / p} \\
& \leq C\left(\int_{0}^{\nu^{2 / 3} / 2}\left(\int_{X}^{\nu^{2 / 3} / 2} \chi_{\left\{X+T>\nu^{-1 / 3}\right\}}(X, T) \frac{|F(-T)|}{T^{2}}\left(\frac{1+X}{1+T}\right)^{1 / 4} d T\right)^{p} d X\right)^{1 / p} \\
& \leq C\left(\int_{0}^{\nu^{2 / 3} / 2}\left(\int_{\max \left\{X, \nu^{-1 / 3}-X\right\}}^{\nu^{2 / 3} / 2} \frac{|F(-T)|}{T^{2}} d T\right)^{p} d X\right)^{1 / p} \\
& \leq C \nu^{-1 /(3 p)}\left(\int_{0}^{\nu^{2 / 3} / 2}\left(\int_{\max \left\{X, 2 \nu^{-1 / 3}-X\right\}}^{\nu^{2 / 3} / 2} T^{-2 p^{\prime}} d T\right)^{p / p^{\prime}} d X\right)^{1 / p} \\
& \times\left(\int_{\nu / 2}^{3 \nu / 2}|f(y)|^{p} d y\right)^{1 / p} .
\end{aligned}
$$

This proves (32) for $i=1$ since

$$
\left(\int_{0}^{\nu^{2 / 3} / 2}\left(\int_{\max \left\{X, \nu^{-1 / 3}-X\right\}}^{\nu^{2 / 3} / 2} T^{-2 p^{\prime}} d T\right)^{p / p^{\prime}} d X\right)^{1 / p} \leq C \nu^{1 / 3} .
$$

Let $i=2$. If $Y \in[-X, 0]$ then $|X-Y|^{-2} \leq C|X|^{-2}$, the change of variable $Y=-T$ and Hölder's inequality similarly show that $I_{2}$ is bounded by $\nu^{-1 /(3 p)}$ multiplied by the product of

$$
\left(\int_{0}^{\nu^{2 / 3} / 2} X^{-2 p}\left(\int_{\max \left\{0, \nu^{-1 / 3}-X\right\}}^{X}\left(\frac{1+X}{1+T}\right)^{p^{\prime} / 4} d T\right)^{p / p^{\prime}} d X\right)^{1 / p}
$$

and the right side of (31). This completes the proof of (32) for $i=2$ once we note that (33) is $O\left(\nu^{1 / 3}\right)$. Consider the case $i=3$. Then $I_{3}$ equals

$$
\left(\int_{\nu^{-1 / 3}}^{\nu^{2 / 3} / 2}\left(\int_{0}^{X / 2} \chi_{\left\{|X-Y|>\nu^{-1 / 3}\right\}}(X, Y) \frac{|F(Y)|}{|X-Y|^{2}}\left(\frac{1+X}{1+Y}\right)^{1 / 4} d Y\right)^{p} d X\right)^{1 / p}
$$


and we further split the $X$-range onto $\left[\nu^{-1 / 3}, 1\right]$ and $\left[1, \nu^{2 / 3} / 2\right]$ denoting the resulting double integrals by $I_{31}$ and $I_{32}$. Then

$$
\begin{aligned}
& I_{31} \leq C\left(\int_{\nu^{-1 / 3}}^{1}\left(\int_{0}^{\min \left\{X / 2, X-\nu^{-1 / 3}\right\}} \frac{|F(Y)|}{X^{2}} d Y\right)^{p} d X\right)^{1 / p} \\
& \leq C\left[\left(\int_{\nu^{-1 / 3}}^{2 \nu^{-1 / 3}}\left(\int_{0}^{X-\nu^{-1 / 3}} \frac{|F(Y)|}{X^{2}} d Y\right)^{p} d X\right)^{1 / p}\right. \\
& \left.+\left(\int_{2 \nu^{-1 / 3}}^{1}\left(\int_{0}^{X / 2} \frac{|F(Y)|}{X^{2}} d Y\right)^{p} d X\right)^{1 / p}\right] \\
& \leq C \nu^{-1 /(3 p)}\left[\left(\int_{\nu^{-1 / 3}}^{2 \nu^{-1 / 3}} X^{-2 p}\left(X-\nu^{-1 / 3}\right)^{p / p^{\prime}} d X\right)^{1 / p}\right. \\
& \left.+\left(\int_{2 \nu^{-1 / 3}}^{1} X^{p\left(1 / p^{\prime}-2\right)} d X\right)^{1 / p}\right]\left(\int_{\nu / 2}^{3 \nu / 2}|f(y)|^{p} d y\right)^{1 / p}
\end{aligned}
$$

where, in the last line, Hölder's inequality was used. Now, the bound

$$
\left(\int_{\nu^{-1 / 3}}^{2 \nu^{-1 / 3}} X^{-2 p}\left(X-\nu^{-1 / 3}\right)^{p / p^{\prime}} d X\right)^{1 / p}+\left(\int_{2 \nu^{-1 / 3}}^{1} X^{p\left(1 / p^{\prime}-2\right)} d X\right)^{1 / p} \leq C \nu^{1 / 3}
$$

shows (32) for $I_{31}$. Similarly, using Hölder's inequality again, we majorize $I_{32}$ by $\nu^{-1 /(3 p)}$ times the product of

$$
\left(\int_{1}^{\nu^{2 / 3} / 2} X^{-7 p / 4}\left(\int_{0}^{X}(1+Y)^{-p^{\prime} / 4} d Y\right)^{p / p^{\prime}} d X\right)^{1 / p}
$$

and the right side of (31). Thus (32) follows for $I_{32}$, hence also for $I_{3}$, since (34) is $O\left(\nu^{1 / 3}\right)$. Consider the case $i=4$. Then $I_{4}$ equals

$$
\left(\int_{0}^{\nu^{2 / 3} / 2}\left(\int_{X / 2}^{2 X} \chi_{\left\{|X-Y|>\nu^{-1 / 3}\right\}}(X, Y) \frac{|F(Y)|}{|X-Y|^{2}}\left(\frac{1+X}{1+Y}\right)^{1 / 4} d Y\right)^{p} d X\right)^{1 / p}
$$

and we further bound it by

$$
\begin{aligned}
& C\left(\int_{0}^{\nu^{2 / 3} / 2}\left(\int_{X / 2}^{2 X} \chi_{\left\{|X-Y|>\nu^{-1 / 3}\right\}}(X, Y) \frac{|F(Y)|}{|X-Y|^{2}} d Y\right)^{p} d X\right)^{1 / p} \\
\leq & C\left(\int_{0}^{\nu^{2 / 3} / 2}\left(\sum_{k=k_{1}}^{\infty} \int_{|X-Y| \sim 2^{k}} \frac{\left|\chi_{\left[0, \nu^{2 / 3} / 2\right]}(Y) F(Y)\right|}{|X-Y|^{2}} d Y\right)^{p} d X\right)^{1 / p} \\
\leq & C\left(\int_{0}^{\nu^{2 / 3} / 2}\left(\sum_{k=k_{1}}^{\infty} 2^{-2 k} \int_{|X-Y| \sim 2^{k}}\left|\chi_{\left[0, \nu^{2 / 3} / 2\right]}(Y) F(Y)\right| d Y\right)^{p} d X\right)^{1 / p} \\
\leq & C\left(\int_{0}^{\nu^{2 / 3} / 2}\left(\sum_{k=k_{1}}^{\infty} 2^{-k} M\left(\chi_{\left[0, \nu^{2 / 3} / 2\right]} F\right)(X)\right)^{p} d X\right)^{1 / p} \\
\leq & C \nu^{1 / 3}\left(\int_{0}^{\nu^{2 / 3} / 2} M\left(\chi_{\left[0, \nu^{2 / 3} / 2\right]} F\right)(X)^{p} d X\right)^{1 / p}
\end{aligned}
$$

with $k_{1}=\left[-\left(\log _{2} \nu\right) / 3\right]-1$. Using the fact that $M$ is a bounded operator on $L^{p}$ we finish the proof of (32) for $i=4$. Consider the case $i=5$. Then $I_{5}$ equals

$$
\left(\int_{0}^{\nu^{2 / 3} / 2}\left(\int_{2 X}^{\nu^{2 / 3} / 2} \chi_{\left\{|X-Y|>\nu^{-1 / 3}\right\}}(X, Y) \frac{|F(Y)|}{|X-Y|^{2}}\left(\frac{1+X}{1+Y}\right)^{1 / 4} d Y\right)^{p} d X\right)^{1 / p}
$$


and is easily seen to be bounded by a constant multiplied by the sum of

$$
I_{51}=\left(\int_{0}^{1}\left(\int_{2 X}^{\nu^{2 / 3} / 2} \chi_{\left\{|X-Y|>\nu^{-1 / 3}\right\}}(X, Y) \frac{|F(Y)|}{Y^{2}} d Y\right)^{p} d X\right)^{1 / p}
$$

and

$$
I_{52}=\left(\int_{1}^{\nu^{2 / 3} / 2}\left(\int_{2 X}^{\nu^{2 / 3} / 2} \chi_{\left\{|X-Y|>\nu^{-1 / 3}\right\}}(X, Y) \frac{|F(Y)|}{Y^{2}} d Y\right)^{p} d X\right)^{1 / p} .
$$

Using Hölder's inequality and the estimate

$$
\left(\int_{0}^{1}\left(\int_{\max \left\{X+\nu^{-1 / 3}, 2 X\right\}}^{\nu^{2 / 3} / 2} Y^{-2} d Y\right)^{p / p^{\prime}} d X\right)^{1 / p} \leq C \nu^{1 / 3}
$$

(decompose the $X$-range into $\left[0, \nu^{-1 / 3}\right]$ and $\left[\nu^{-1 / 3}, 1\right]$ ) shows that

$$
\begin{aligned}
I_{51} & \leq C\left(\int_{0}^{1}\left(\int_{\max \left\{X+\nu^{-1 / 3}, 2 X\right\}}^{\nu^{2 / 3} / 2} \frac{|F(Y)|}{Y^{2}} d Y\right)^{p} d X\right)^{1 / p} \\
& \leq C \nu^{1 / 3-1 /(3 p)}\left(\int_{\nu / 2}^{3 \nu / 2}|f(y)|^{p} d y\right)^{1 / p} .
\end{aligned}
$$

Similarly, the estimate

$$
\left(\int_{1}^{\nu^{2 / 3} / 2}\left(\int_{2 X}^{\nu^{2 / 3} / 2} Y^{-2 p^{\prime}} d Y\right)^{p / p^{\prime}} d X\right)^{1 / p} \leq C \nu^{1 / 3}
$$

and Hölder's inequality produce

$$
\begin{aligned}
I_{52} & \leq C\left(\int_{1}^{\nu^{2 / 3} / 2}\left(\int_{2 X}^{3 \nu^{2 / 3}} Y^{-2 p^{\prime}} d Y\right)^{p / p^{\prime}} d X\right)^{1 / p}\left(\int_{-\nu^{2 / 3} / 2}^{\nu^{2 / 3} / 2}|F(Y)|^{p} d Y\right)^{1 / p} \\
& \leq C \nu^{1 / 3-1 /(3 p)}\left(\int_{\nu / 2}^{3 \nu / 2}|f(y)|^{p} d y\right)^{1 / p} .
\end{aligned}
$$

This finishes the proof of (32) for $i=5$ hence the proposition.

Lemma 4. Let

$$
\begin{aligned}
& R_{1}=[2 \bar{\nu} / 3, \infty) \times[0, \bar{\nu} / 2], \quad R_{2}=[3 \bar{\nu} / 2, \infty) \times[\bar{\nu} / 2,5 \bar{\nu} / 4], \\
& R_{3}=[0, \bar{\nu} / 2] \times[2 \bar{\nu} / 3, \infty), \quad R_{4}=[\bar{\nu} / 2,5 \bar{\nu} / 4] \times[3 \bar{\nu} / 2, \infty) .
\end{aligned}
$$

Then there exists a constant $C$ independent of $\nu>-1$ such that

$$
\left|K_{\nu}^{1}(x, y) \chi_{R_{i}}(x, y)\right| \leq C \frac{\Phi_{\nu}(x) \Phi_{\nu}(y)}{|x-y|^{2}}, \quad i=1,2,3,4 .
$$

Proof. We discuss the case $\nu \geq 1$ only (if $\nu<1$ our argument also applies but the right side of (29) must be suitably modified). It is sufficient to check that the expression in parentheses on the right side of (29) divided by $(x+y)^{2}$ is bounded by a constant. In $R_{1}, x \leq x+y \leq 7 x / 4$ and $x / 4 \leq x-y \leq x$, therefore $v /|u|$ is bounded. Also each of the terms $\left(\left|\nu^{2}-x^{2}\right|+x^{4 / 3}\right)^{1 / 2},\left(\left|\nu^{2}-y^{2}\right|+y^{4 / 3}\right)^{1 / 2}$, when divided by $x+y$ is bounded. Finally, $\left|2 \nu^{2}-\left(x^{2}+y^{2}\right)\right|$ when divided by $(x+y)^{2}$ is bounded. Similar analysis holds for $i=2,3,4$.

Proposition 4. Let $\alpha>-1,1 \leq p \leq \infty$ and $a, A, b, B$ satisfy the assumptions (3) and (4) of Theorem 1. Assume also that

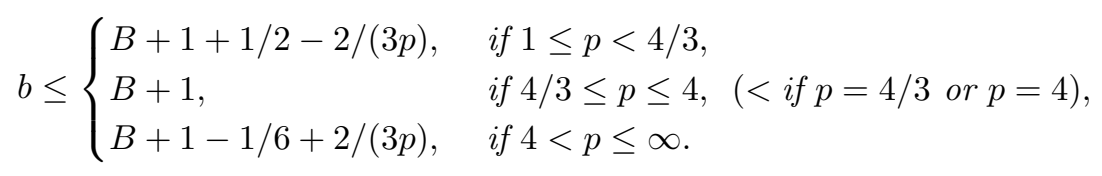


Then, for $\nu \geq \alpha$,

(35)

$$
\int_{0}^{\infty}\left(w_{a, b}(x) \int_{0}^{\infty} \chi_{R_{i}}(x, y)\left|K_{\nu}^{1}(x, y) f(y)\right| d y\right)^{p} d x \leq C \int_{0}^{\infty}\left|f(y) w_{A, B}(y)\right|^{p} d y,
$$

where $R_{i}, i=1, \ldots, 4$, are as in Lemma 4 .

Proof. Denote the left side of the inequality to be proved raised to the $1 / p$ th power (with the usual interpretation when $p=\infty$ ) by $F_{i}=F_{i}(a, b, p, \nu ; f)$. Consider first the case $i=1$. Using the bound from Lemma 4 and Hölder's inequality gives

$$
\begin{aligned}
F_{1} & =\left(\int_{0}^{\infty}\left(w_{a, b}(x) \int_{0}^{\infty} \chi_{R_{1}}(x, y)\left|K_{\nu}^{1}(x, y) f(y)\right| d y\right)^{p} d x\right)^{1 / p} \\
& \leq C\left(\int_{2 \bar{\nu} / 3}^{\infty}\left(x^{b} \int_{0}^{\bar{\nu} / 2}|f(y)| \frac{\Phi_{\nu}(x) \Phi_{\nu}(y)}{|x-y|^{2}} d y\right)^{p} d x\right)^{1 / p} \\
& \leq C\left(\int_{2 \bar{\nu} / 3}^{\infty}\left(x^{b-2} \Phi_{\nu}(x) \int_{0}^{\bar{\nu} / 2}|f(y)| \Phi_{\nu}(y) d y\right)^{p} d x\right)^{1 / p} \\
& \leq C\left\|\chi_{(2 \bar{\nu} / 3, \infty)}(x) x^{b-2} \Phi_{\nu}(x)\right\|_{p} \cdot\left\|\chi_{(0, \bar{\nu} / 2)}(y) w_{A, B}(y)^{-1} \Phi_{\nu}(y)\right\|_{p^{\prime}} \cdot\left\|f(y) w_{A, B}(y)\right\|_{p} .
\end{aligned}
$$

The product of the first two terms following the last $C$ above is a continuous function of $\nu \in[\alpha, \infty)$. It is therefore sufficient to consider large $\nu$ 's; analogous remark applies when analysing $F_{i}, i=2,3,4$. Thus, from now on we assume $\nu$ to be large and write $\nu$ in place of $\bar{\nu}$. Then we have

$$
\begin{aligned}
& \left\|\chi_{(0, \nu / 2)}(y) w_{A, B}(y)^{-1} \Phi_{\nu}(y)\right\|_{p^{\prime}} \\
\leq & C \max \left\{\left\|\chi_{(0,1)}(y) y^{-A} \Phi_{\nu}(y)\right\|_{p^{\prime}},\left\|\chi_{(1, \nu / 2)}(y) y^{-B} \Phi_{\nu}(y)\right\|_{p^{\prime}}\right\} \\
\leq & C 2^{-\nu} \Gamma(\nu+1)^{-1}(\nu / 2)^{-B+\nu+1 / 2},
\end{aligned}
$$

where, to assure the convergence of the integral on $[0,1]$ the condition $A<1-$ $1 / p+(\alpha+1 / 2)$ was used $(A \leq \alpha+1 / 2$ when $p=1)$. Moreover,

$$
\left\|\chi_{(2 \nu / 3, \infty)}(x) x^{b-2} \Phi_{\nu}(x)\right\|_{p} \leq C \nu^{b-2+1 / p} \begin{cases}1, & \text { if } p<4 \\ (\log \nu)^{1 / 4}, & \text { if } p=4 \\ \nu^{1 / 6-2 /(3 p)}, & \text { if } p>4\end{cases}
$$

This is obtained by splitting the integration onto $(2 \nu / 3,2 \nu)$ and $(2 \nu, \infty)$, using the assumption $b<2-1 / p(b \leq 2$ when $p=\infty)$ and then by inspection. We conclude the analysis by noting that the product of majorants in (36) and (37) produces a bounded function of $\nu \geq 1$.

In the case $i=2$, to get the required bound we proceed as in the case just discussed majorizing $F_{2}$ by a constant times

$$
\left\|\chi_{(3 \nu / 2, \infty)}(x) x^{b-2}\right\|_{p} \cdot\left\|\chi_{(\nu / 2,5 \nu / 4)}(y) w_{A, B}(y)^{-1} \Phi_{\nu}(y)\right\|_{p^{\prime}} \cdot\left\|f(y) w_{A, B}(y)\right\|_{p} .
$$

Again, we claim that the product of the first two terms above is a bounded function of $\nu \geq 1$. This is because $\left\|\chi_{(3 \nu / 2, \infty)}(x) x^{b-2}\right\|_{p}=O\left(\nu^{b-2+1 / p}\right)$ and

$$
\left\|\chi_{(\nu / 2,5 \nu / 4)}(y) w_{A, B}(y)^{-1} \Phi_{\nu}(y)\right\|_{p^{\prime}} \leq C \nu^{-B+1-1 / p} \begin{cases}\nu^{-1 / 2+2 /(3 p)}, & \text { if } p<4 / 3 \\ (\log \nu)^{1 / 4}, & \text { if } p=4 / 3 \\ 1, & \text { if } p>4 / 3 .\end{cases}
$$

Now, considering separately the cases $p<4 / 3, p=4 / 3, p>4 / 3$ and using appropriate assumptions on the relation between $b$ and $B$ proves the claim.

Consider the case $i=3$. This time we bound $F_{3}$ by a constant times

$$
\left\|\chi_{(0, \nu / 2)}(x) w_{a, b}(x) \Phi_{\nu}(x)\right\|_{p} \cdot\left\|\chi_{(2 \nu / 3, \infty)}(y) y^{-(B+2)} \Phi_{\nu}(y)\right\|_{p^{\prime}} \cdot\left\|f(y) w_{A, B}(y)\right\|_{p}
$$


Similarly to (36) (the assumption $a>-1 / p-(\alpha+1 / 2)$, with $a \geq-(\alpha+1 / 2)$ when $p=\infty$, is used), we obtain the estimate

$$
\left\|\chi_{(0, \nu / 2)}(x) w_{a, b}(x) \Phi_{\nu}(x)\right\|_{p} \leq C 2^{-\nu} \Gamma(\nu+1)^{-1}(\nu / 2)^{b+\nu+1 / 2} .
$$

Moreover,

$$
\left\|\chi_{(2 \nu / 3, \infty)}(y) y^{-(B+2)} \Phi_{\nu}(y)\right\|_{p^{\prime}} \leq C \nu^{-B-1-1 / p} \begin{cases}\nu^{-1 / 2+2 /(3 p)}, & \text { if } p<4 / 3 \\ (\log \nu)^{1 / 4}, & \text { if } p=4 / 3 \\ 1, & \text { if } p>4 / 3 .\end{cases}
$$

This is easily seen by splitting the integration onto $(2 \nu / 3,2 \nu)$ and $(2 \nu, \infty)$, using the relevant assumption and then by inspection. Combining these two bounds shows that the product of the first two terms majorizing $F_{3}$ is a bounded function of $\nu \geq 1$.

Finally, consider $i=4$. Then $F_{4}$ is bounded by a constant times

$$
\left\|\chi_{(\nu / 2,5 \nu / 4)}(x) x^{b} \Phi_{\nu}(x)\right\|_{p} \cdot\left\|\chi_{(3 \nu / 2, \infty)}(y) y^{-(B+2)}\right\|_{p^{\prime}} \cdot\left\|f(y) w_{A, B}(y)\right\|_{p} .
$$

Here we use $\left\|\chi_{(3 \nu / 2, \infty)}(y) y^{-(B+2)}\right\|_{p^{\prime}}=O\left(\nu^{-B-1-1 / p}\right)$ (the assumption $B>-1-$ $1 / p, B \geq-2$ when $p=1$, is applied) and (37) with $b$ instead of $b-2$ (to be precise with $(\nu / 2,5 \nu / 4)$ in place of $(2 \nu / 3, \infty))$. Then, considering separately the cases $p<4 / 3, p=4 / 3, p>4 / 3$ and using appropriate assumptions on the relation between $b$ and $B$ shows that the product of the two relevant terms is a bounded function of $\nu \geq 1$.

\section{Proof of Theorem 1}

We start with the proof of sufficiency in Theorem 1 . If $\alpha<1$ and $\nu \in[\alpha, 1]$, then (2) is a consequence of the estimates contained in Propositions 1, 2 and 4 . If $\alpha \geq 1$ and $\nu \in[\alpha, \infty)$, then we use the estimates from Propositions 1-4 (note that the regions that appear there cover the quarter plane $(0, \infty) \times(0, \infty))$. Checking the required assumptions shows that $(3),(4)$ and (5) were used.

We now pass to the necessity. Checking of (3) relies on the asymptotics of $K_{\alpha}^{1}(x, y)$ at $(0,0)$. Indeed, to check the necessity of the first condition in (3) note that for a sufficiently small $\varepsilon=\varepsilon(\alpha)$ we have $J_{\alpha}(s) \geq C s^{\alpha}$ for $0<s<\varepsilon$. Hence, for $x, y \in(0, \varepsilon), K_{\alpha}^{1}(x, y) \geq C(x y)^{\alpha+1 / 2}$. Take $f=\chi_{(\varepsilon / 2, \varepsilon)}$; then, for $x \in(0, \varepsilon)$,

$$
S_{\alpha}^{1} f(x)=\int_{0}^{\infty} K_{\alpha}^{1}(x, y) f(y) d y \geq C x^{\alpha+1 / 2} \int_{\varepsilon / 2}^{\varepsilon} y^{\alpha+1 / 2} d y \geq C x^{\alpha+1 / 2} .
$$

Thus, (here and later on an appropriate interpretation is needed if $p=\infty$ ), by (2),

$$
\begin{aligned}
\left(\int_{0}^{\varepsilon} x^{(\alpha+1 / 2+a) p} d x\right)^{1 / p} & \leq C\left(\int_{0}^{\varepsilon}\left|S_{\alpha}^{1} f(x) x^{a}(1+x)^{b-a}\right|^{p} d x\right)^{1 / p} \\
& \leq C\left(\int_{\varepsilon / 2}^{\varepsilon}\left|x^{A}(1+x)^{B-A}\right|^{p} d x\right)^{1 / p} \\
& <\infty
\end{aligned}
$$

In consequence, $a>-1 / p-(\alpha+1 / 2)(\geq$ if $p=\infty)$ follows.

To verify the necessity of the second part of (3) define the function $f$ by

$$
f(x)= \begin{cases}x^{-1-A} /(-\log x)^{2}, & \text { if } p=1 \\ x^{-1 / p-A} /(-\log x), & \text { if } 1<p<\infty \\ x^{-A}, & \text { if } p=\infty\end{cases}
$$


for $0<x<\varepsilon$ and $f(x)=0$ otherwise, and consider in (2) the sequence of functions $f_{n}(x)=f(x) \chi_{(\varepsilon / n, \varepsilon)}(x)$. Then, $\sup _{n \geq 1}\left\|f_{n}(x) x^{A}(1+x)^{B-A}\right\|_{p}<\infty$ and, for $0<$ $x<\varepsilon$,

$$
S_{\alpha}^{1} f_{n}(x)=\int_{0}^{\infty} K_{\alpha}^{1}(x, y) f_{n}(y) d y \geq C x^{\alpha+1 / 2} \int_{\varepsilon / n}^{\varepsilon} y^{\alpha+1 / 2} f(y) d y .
$$

Thus, to keep $\left\|S_{\alpha}^{1} f_{n}(x) x^{a}(1+x)^{b-a}\right\|_{p}$ uniformly bounded (with respect to $n$ ) requires $\alpha+1 / 2-A-1 / p>-1$ ( $\geq$ if $p=1$ ), the second part of (3).

To check the necessity of the first condition in (4) choose a sufficiently small $\varepsilon$ such that $J_{\alpha}(y)>0$ for $0<y<\varepsilon$ and take $f=\chi_{(\varepsilon / 2, \varepsilon)}$. Then, $S_{\alpha}^{1} f(x)$ is the sum of

$$
\begin{aligned}
& 2 \int_{\varepsilon / 2}^{\varepsilon} \frac{2 \alpha^{2}-\left(x^{2}+y^{2}\right)}{\left(x^{2}-y^{2}\right)^{2}} y^{1 / 2} J_{\alpha}(y) d y \cdot x^{1 / 2} J_{\alpha}(x), \\
& -4 x \int_{\varepsilon / 2}^{\varepsilon} \frac{x^{2}+y^{2}}{\left(x^{2}-y^{2}\right)^{3}} y^{1 / 2} J_{\alpha}(y) d y \cdot x^{1 / 2} J_{\alpha}^{\prime}(x), \\
& \quad 4 \int_{\varepsilon / 2}^{\varepsilon} \frac{y\left(x^{2}+y^{2}\right)}{\left(x^{2}-y^{2}\right)^{3}} y^{1 / 2} J_{\alpha}^{\prime}(y) d y \cdot x^{1 / 2} J_{\alpha}(x), \\
& -4 x \int_{\varepsilon / 2}^{\varepsilon} \frac{y}{\left(x^{2}-y^{2}\right)^{2}} y^{1 / 2} J_{\alpha}^{\prime}(y) d y \cdot x^{1 / 2} J_{\alpha}^{\prime}(x) .
\end{aligned}
$$

Since

$$
x^{1 / 2} J_{\alpha}(x)=(2 / \pi)^{1 / 2} \cos \left(x-\frac{\alpha \pi}{2}-\frac{\pi}{4}\right)+O\left(x^{-1}\right), \quad x \geq 1,
$$

and

hence

$$
J_{\alpha}^{\prime}(x)=\frac{\alpha}{x} J_{\alpha}(x)-J_{\alpha+1}(x)
$$

$$
x^{1 / 2} J_{\alpha}^{\prime}(x)=(2 / \pi)^{1 / 2} \sin \left(x-\frac{\alpha \pi}{2}-\frac{\pi}{4}\right)+O\left(x^{-1}\right), \quad x \geq 1 .
$$

Let $g$ and $h, 4<g<h<\infty$, be such that $x^{1 / 2} J_{\alpha}(x) \geq 1 / \sqrt{2 \pi}$ for $x \in X=$ $\cup_{j=0}^{\infty}(g+2 \pi j, h+2 \pi j)$. Then, for $x \in X$, the absolute values of (39), (40) and (41) are less than $C x^{-3}$, while the (negative) value of (38) is less than $-C x^{-2}$. Thus, for large values of $x \in X$, the sum of absolute values of (39), (40) and (41) is less than the half of the absolute value of (38). Therefore, it is possible to choose $g$ so large that the former statement remains valid for all $x \in X$. Hence, with such a choice of $g$, for $x \in X,\left|S_{\alpha}^{1} f(x)\right| \geq C x^{-2}$, and using (2) produces

$$
\begin{aligned}
\left(\int_{X} x^{(b-2) p} d x\right)^{1 / p} & \leq C\left(\int_{X}\left|S_{\alpha}^{1} f(x) x^{a}(1+x)^{b-a}\right|^{p} d x\right)^{1 / p} \\
& \leq C\left(\int_{\varepsilon / 2}^{\varepsilon}\left|f(x) x^{A}(1+x)^{B-A}\right|^{p} d x\right)^{1 / p} \\
& <\infty .
\end{aligned}
$$

In consequence, $b<2-1 / p(\leq$ if $p=\infty)$ follows.

To check the necessity of the second condition in (4), define the function $f$ by

$$
f(x)= \begin{cases}x^{-1-B} /(\log x)^{2}, & \text { if } p=1, \\ x^{-1 / p-B} /(\log x), & \text { if } 1<p<\infty, \\ x^{-B}, & \text { if } p=\infty,\end{cases}
$$

for $x \geq 2$ and $f(x)=0$ otherwise. Then choose an interval $(g, h), 1<g<h<\infty$, such that $J_{\alpha}(x)<0$ and $J_{\alpha}^{\prime}(x)>0$ for $x \in(g, h)$. In addition, choose $G$ and $H$, 
$2 \max \{g, \alpha\}<G<H<\infty$ in such a way that $y^{1 / 2} J_{\alpha}(y) \geq 1 / \sqrt{2 \pi}$ and $J_{\alpha}^{\prime}(y) \leq 0$ for $y \in Y=\cup_{j=0}^{\infty}(G+2 \pi j, H+2 \pi j)$. Consider in (2) the sequence of functions $f_{n}(x)=f(x) \chi_{Y_{n}}(x), Y_{n}=Y \cap(2, n)$. Then, $\sup _{n \geq 1}\left\|f_{n}(x) x^{A}(1+x)^{B-A}\right\|_{p}<\infty$ and $S_{\alpha}^{1} f_{n}(x)$ is the sum of

$$
\begin{aligned}
& I_{1}(n, x)=2 \int_{Y_{n}} f(y) \frac{2 \alpha^{2}-\left(x^{2}+y^{2}\right)}{\left(x^{2}-y^{2}\right)^{2}} y^{1 / 2} J_{\alpha}(y) d y \cdot x^{1 / 2} J_{\alpha}(x), \\
& I_{2}(n, x)=-4 x \int_{Y_{n}} f(y) \frac{x^{2}+y^{2}}{\left(x^{2}-y^{2}\right)^{3}} y^{1 / 2} J_{\alpha}(y) d y \cdot x^{1 / 2} J_{\alpha}^{\prime}(x), \\
& I_{3}(n, x)=4 \int_{Y_{n}} f(y) \frac{y\left(x^{2}+y^{2}\right)}{\left(x^{2}-y^{2}\right)^{3}} y^{1 / 2} J_{\alpha}^{\prime}(y) d y \cdot x^{1 / 2} J_{\alpha}(x), \\
& I_{4}(n, x)=-4 x \int_{Y_{n}} f(y) \frac{y}{\left(x^{2}-y^{2}\right)^{2}} y^{1 / 2} J_{\alpha}^{\prime}(y) d y \cdot x^{1 / 2} J_{\alpha}^{\prime}(x) .
\end{aligned}
$$

The choice of $f,(g, h)$ and $Y$ shows that for $x \in(g, h), I_{1}(n, x), I_{2}(n, x)$ and $I_{4}(n, x)$ are positive while $I_{3}(n, x)$ in negative. Thus $I_{1}(n, x)+I_{3}(n, x) \leq S_{\alpha}^{1} f_{n}(x)$. Moreover,

$$
0<\int_{Y_{n}} \frac{f(y)}{y^{2}} d y \cdot\left(-x^{1 / 2} J_{\alpha}(x)\right) \leq C I_{1}(n, x)
$$

and

$$
\left|I_{3}(n, x)\right| \leq C \int_{Y_{n}} \frac{f(y)}{y^{3}} d y \cdot\left(-x^{1 / 2} J_{\alpha}(x)\right) .
$$

The last estimate shows that $G$ might be chosen so large that $\left|I_{3}(n, x)\right| \leq 10^{-2} I_{1}(n, x)$ for $x \in(g, h)$. With such a choice of $G$,

$$
\int_{Y_{n}} \frac{f(y)}{y^{2}} d y \cdot\left(-x^{1 / 2} J_{\alpha}(x)\right) \leq C S_{\alpha}^{1} f_{n}(x)
$$

and then multiplication of both sides by $x^{b}$ and $x$-integration over $(g, h)$ produces

$$
\begin{aligned}
\int_{Y_{n}} \frac{f(y)}{y^{2}} d y \cdot\left(\int_{g}^{h}\left|J_{\alpha}(x) x^{(b+1 / 2)}\right|^{p} d x\right)^{1 / p} & \leq C\left(\int_{g}^{h}\left|S_{\alpha}^{1} f_{n}(y) y^{b}\right|^{p} d y\right)^{1 / p} \\
& \leq C\left\|f_{n}(x) x^{A}(1+x)^{B-A}\right\|_{p} .
\end{aligned}
$$

Thus, uniform boundedness (with respect to $n$ ) of the integrals $\int_{Y_{n}} f(y) y^{-2} d y$ now requires $B>-1-1 / p(\geq$ if $p=1)$, the second part of (4).

Proving the necessity of (5) note that for $R>0, K_{\alpha, R}^{1}(x, y)=R K_{\alpha, 1}^{1}(x R, y R)$, hence $S_{\alpha, R}^{1} f(x)=S_{\alpha, 1}^{1} f_{R}(x R)$, where $f_{R}(y)=f(y / R)$. Then, applying (2) (where $R=1$ is assumed) to a nontrivial function $f \in C_{c}^{\infty}(0, \infty)$ produces

$$
\begin{aligned}
\left\|S_{\alpha, R}^{1} f(x) x^{a}\left(R^{-1}+x\right)^{b-a}\right\|_{p} & \left.\leq C R^{B-b} \| f(x) x^{A}\left(R^{-1}+x\right)^{B-A}\right) \|_{p} \\
& \leq C R^{B-b}\left\|f(x) x^{B}\right\|_{p}
\end{aligned}
$$

if $B-A \leq 0$ or with the last term replaced by $\left\|f(x) x^{A}(1+x)^{B-A}\right\|_{p}$ if $B-A>0$ and $R>1$. But

$$
\lim _{R \rightarrow \infty} S_{\alpha, R}^{1} f(x)=f(x), \quad x>0,
$$

hence by (42), (43) and Fatou's lemma for $p<\infty$ or trivially for $p=\infty$ we have

$$
\left\|f(x) x^{b}\right\|_{p} \leq C\left\|f(x) x^{B}\right\|_{p} \liminf _{R \rightarrow \infty} R^{B-b}
$$

if $B-A \leq 0$ or analogous inequality with $x^{B}$ replaced by $x^{A}(1+x)^{B-A}$ when $B-A>0$. In any case, this implies $b \leq B$. To verify (43), fix $x>0$ and 
$f \in C_{c}^{\infty}(0, \infty)$ and use $S_{\nu, R}^{1} f(x)=\mathcal{H}_{\nu}\left(m_{R}^{1} \cdot \mathcal{H}_{\nu} f\right)(x)$ to write

$$
S_{\nu, R}^{1} f(x)=\int_{0}^{\infty}(x y)^{1 / 2} J_{\alpha}(x y)\left(1-\left(\frac{y}{R}\right)^{2}\right)_{+} \mathcal{H}_{\alpha} f(y) d y .
$$

Then, (43) follows by an application of the dominated convergence theorem and the inversion formula for the Hankel transform $\mathcal{H}_{\alpha}$ once we note that

$$
\int_{0}^{\infty}(x y)^{1 / 2}\left|J_{\alpha}(x y) \| \mathcal{H}_{\alpha} f(y)\right| d y<\infty .
$$

The above is a consequence of $\mathcal{H}_{\alpha} f(y)=O\left(y^{\alpha+1 / 2}\right), y \rightarrow 0^{+}$, which in turn follows from (6) and $\mathcal{H}_{\alpha} f(y)=O\left(y^{-2}\right)$, the latter easily implied by (22). This finishes the proof of the necessity of (5), hence the theorem.

\section{REFERENCES}

[1] J. A. Barceló and A. Córdoba, Band-limited functions: $L^{p}$-convergence, Trans. Amer. Math. Soc. 313 (1989), 655-669.

[2] A. Córdoba, The disc multiplier, Duke Math. J. 58 (1989), 21-27.

[3] N. N. Lebedev, Special functions and its applications, Dover, New York, 1972.

[4] B. Muckenhoupt, Mean convergence of Hermite and Laguerre series. I, Trans. Amer. Math. Soc. 147 (1970), 419-431; II, ibid. 147 (1970), 433-460.

[5] B. Muckenhoupt and D. W. Webb, Two-weight norm inequalities for Cesàro means of Laguerre expansions, Trans. Amer. Math. Soc. 353 (2000), 1119-1149.

[6] E. L. Poiani, Mean Cesàro summability of Laguerre and Hermite series, Trans. Amer. Math. Soc. 173 (1972), 1-31.

[7] K. Stempak, Uniform two-weight norm inequalities for Hankel transform partial sum operators, Houston J. Math. 29 (2003), 1045-1063.

[8] D. W. Webb, Pointwise estimates of the moduli of Cesàro-Laguerre kernels, preprint.

Departamento de Matemáticas y Computación, Universidad de La Rioja, Calle Luis DE UlloA S/N, 26004 Logroño, SPAin

E-mail address: oscar.ciaurri@dmc.unirioja.es

Instytut Matematyki, Politechnika Wroceawska, Wyb. Wyspiańskiego 27, 50-370 Wroceaw, Poland

E-mail address: stempak@im.pwr.wroc.pl

Departamento de Matemáticas y Computación, Universidad de La Rioja, Calle Luis DE Ulloa S/N, 26004 LOGROÑo, SPAin

E-mail address: jvarona@dmc.unirioja.es 